PACS: $73.40 . \mathrm{Lq}$

\title{
Obtaining heterostructures with quantium dots for sensors by using liquid phase epitaxy
}

\author{
I.E. Maronchuk ${ }^{1}$, A.M. D’yachenko ${ }^{2}$, A.I. Minailov ${ }^{1}$, V.V. Kurak ${ }^{1}$, I.V. Chorny ${ }^{1}$ \\ 1 Kherson State Technical University, 24, Berislavskoye Shosse, 73008 Kherson, Ukraine \\ E-mail:design@tlc.kherson.ua,andymin@selena.net.ua \\ 2 Mechnikov Odessa National University, 2, Dvoryanskaya str., 65026 Odessa, Ukraine
}

\begin{abstract}
Peculiarities of the crystallization processes forming the structures with quantum dots (QD) by the method of pulse cooling of saturated solution-melt were considered. A theoretical model of QD formation was developed, and it was shown that sizes of experimentally obtained QDs are in good accordance with those theoretically calculated taking into account the Rehbinder effect and the process of nuclei increase. The STM-image and the photoluminescence spectra of the structures with InAs QD grown on GaAs substrate and also InSb quantum dots grown on $\mathrm{GaSb}$ substrate are represented.
\end{abstract}

Keywords: liquid phase epitaxy, quantum dots, nucleus, supersaturation, strain.

Manuscript received 26.07.04; accepted for publication 16.12.04.

\section{Introduction}

Quantum dots (QDs) attract an increasing attention of researchers in view of the perspectives to create different nanoelectronic devices on their base. The electronic processes in these structures should not be described using conceptions about the gas of quasiparticles, which is used for the description of electronic processes in solids, but the technology of obtaining the structures with regularly located quantum-size dots requires a development of new non-standard, selforganizing processes that are not used in the technology of crystals and epitaxial structures [1].

At the present time, the basic technique to obtain QD structures are MBE and MOCVD, in which the formation of QDs was realized by the Stranski Krastanov mechanism, while using the substrates with the lattice constant possessing an essential difference from the lattice constant of crystallized quantum dot material [2].

Though a considerable progress in the technology of QD preparation by using these methods, the high cost of the technological equipment and specific defects in structures obtained by MBE and MOCVD technology require searching some alternative methods suitable to produce definite types of low-dimensional structures and may be enough progressive and economically justified in competition with those used today. Alternatively, this work consider the opportunity of using the method of pulse cooling of saturated solutionmelt for obtaining epitaxial low-dimensional heterostructures with QDs [3].

\section{Experimental}

The growth of InAs QDs was realized from indium solution-melts, and formation of GaAs matrix layers from $\mathrm{Ga}, \mathrm{Ga}+\mathrm{In}$ or In melts in slider-type cassette (Fig. 1).

Growth temperature $T_{1}$ was varied within the range 300 to $500{ }^{\circ} \mathrm{C}$. The temperature of the heat-absorber $T_{P}$ was selected in such a manner that the supercooling $\Delta T_{F}$ at the crystallization front did not exceed $5 \ldots 7^{\circ} \mathrm{C}$ to avoid homogeneous nucleation in the liquid phase. GaAs wafers with surface orientation (100) and GaAs wafers disoriented from $(100)$ by $4^{\circ}$ in direction $\langle 110\rangle$ were used as substrates.

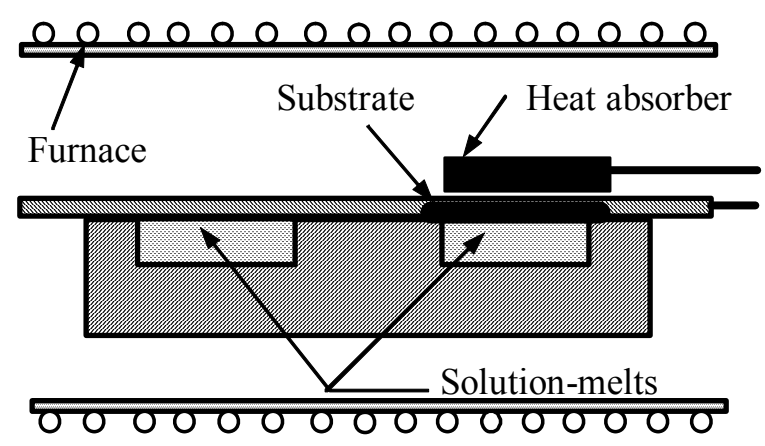

Fig. 1. Schematic sketch of growth cassette for growing heterostructures with QDs. 
Growing these heterostructures containing the QDs by pulse cooling of solution-melt was realized as follows [3]: a substrate at the temperature $T_{1}$ was brought into contact with solution-melt saturated at this temperature. Then, the heat-absorber with thickness $\delta$ having a temperature $T_{P}$ that was less than the substrate temperature by the value of $\Delta T=T_{1}-T_{P}$ was placed on the substrate backside. After some time $\tau$, the heatabsorber was heated up to the temperature $T_{1}$. During this period, cooling the substrate and layer of the solution-melt near its surface was occurred. It resulted in growing the epitaxial layer.

The characterization of the structures obtained was realized using the photoluminescence technique (PL) and scanning tunnel microscopy (STM). Photoluminescence (PL) spectra were measured at 300 and $77 \mathrm{~K}$ using a diffraction grating with ruling 300 lines/mm. PL was excited by a semiconductor laser having power $5 \mathrm{~mW}$, by helium-neon laser, power $P_{\max }=20 \mathrm{~mW}$ and by argon laser with the power up to $5 \mathrm{~W}$. A germanium photodiode and a photomultiplier with spectral response in longwave area up to $1200 \mathrm{~nm}$ were used as photodetectors. Investigated PL emission was extracted both from a surface of a sample and from its butt end. In the last case, the samples obtained were split into parallelepipeds with width 1 to $2 \mathrm{~mm}$ and length 10 to $15 \mathrm{~mm}$. A laser beam was directed at the sample surface, and the PL emergent from the butt end of the sample was directed at the monochromator entrance slit.

For the given values of the process temperature $T_{1}$, material of the heat-absorber and its initial temperature $T_{P}$, and thickness $\tau$, thickness and material of substrate, thickness and material of solution-melt with using nonstationary thermal conductivity equation, the temperature distribution in the system heat-absorber substrate - solution-melt and its temporal change were calculated under the assumption of a direct contact of the heat-absorber and the substrate. Then taking into account that minimum overcooling at crystallization front, which is necessary for growing the epitaxial layer, makes $0.025^{\circ} \mathrm{C}[3]$, the cooling pulse duration $\tau$ and average width of crystallized layer $h$ have been determined (Table 1).

Table 1. The values $\tau$ and $h$ at the growth of epitaxial layers InAs at $T_{1}=400{ }^{\circ} \mathrm{C}$.

\begin{tabular}{|c|c|c|c|c|c|c|}
\hline \multirow{2}{*}{$\begin{array}{c}\Delta T_{F} \\
(\mathrm{C})\end{array}$} & \multicolumn{2}{|c|}{$\delta=0.1 \mathrm{~cm}$} & \multicolumn{2}{c|}{$\delta=0.3 \mathrm{~cm}$} & \multicolumn{2}{c|}{$\delta=0.5 \mathrm{~cm}$} \\
\cline { 2 - 7 } & $\tau(\mathrm{s})$ & $h(\mathrm{~nm})$ & $\tau(\mathrm{s})$ & $h(\mathrm{~nm})$ & $\tau(\mathrm{s})$ & $h(\mathrm{~nm})$ \\
\hline 2 & 0.028 & 0.93 & 0.100 & 1.76 & 0.720 & 4.73 \\
\hline 5 & 0.037 & 2.60 & 0.122 & 4.72 & 0.895 & 12.80 \\
\hline 10 & 0.045 & 5.46 & 0.140 & 9.64 & 1.025 & 26.08 \\
\hline
\end{tabular}

As it follows from Table 1, the layers having uniform thickness may be obtained either at a large magnitude of $\Delta T_{F}$ and small thickness of the heatabsorber $\delta$, or at a small $\Delta T_{F}$ and large value $\delta$. At the first case, the rate of the supersaturation increase and temperature gradient in the solution-melt will be higher, and duration of a cooling pulse relaxation $\tau$ will be less than in the second case. The magnitude of supersaturation increase rate and cooling pulse relaxation duration have an influence on non-stationary processes of QD formation and determine a ratio between heterogeneous and homogeneous crystallization.

\section{The analysis of growing processes}

The driving force of the crystallization process is the difference of chemical potentials of atoms of crystallizing material in liquid $\left(\mu_{L}\right)$ and solid $\left(\mu_{S}\right)$ phases. As the lattice constant of a material of a QD $a_{2}$ essentially differs from a lattice constant of a substrate material $a_{1}$, the shear strain arises at heterointerface with substrate during the formation of QDs. This mechanical strain may be expressed through the elastic energy $U(r)$ per atom of a QD at heterointerface. Then, the difference of chemical potentials is determined by the following expression:

$\Delta \mu_{S T}=\mu_{L}-\mu_{S}-U(r)=\Delta \mu-U(r)$,

where

$\Delta \mu=k T \ln \frac{C}{C_{0}}$,

$C, C_{0}-$ concentrations of crystallizing matter in oversaturated solution and in equilibrium solution, correspondingly; $k$ - Boltzmann constant [4];

$U(r)=\frac{G L^{2}}{4 N_{S} a_{0}}$,

$N_{S}$ - number of atoms per unit of surface area; $a_{0}-$ interatomic space of the fixed lattice; $L$ - relative atomic displacement:

$L=\frac{2\left(a_{1}-a_{2}\right)}{a_{1}+a_{2}} r+l_{a_{1}}-l_{a_{2}}$,

$l_{a_{1}}, l_{a_{2}}$ - elastic displacements of atoms in materials of QDs and substrates, correspondingly; $G$ - modulus of shearing in a QD layer at heterointerface [5]:

$G=\frac{4 \pi \sigma}{a_{0}}$,

$\sigma \quad$ - interphase surface energy using the Rehbinder effect.

The interphase surface energy at the contact of the solid phase with the liquid phase using the Rehbinder effect is determined by the expression: 
$\sigma \leq \sigma_{A} \frac{Q_{m A}}{Q_{v A}}(1-y)+\sigma_{B} \frac{Q_{m B}}{Q_{v B}} y+\frac{N_{S} z_{b}}{z} U_{0}(y-x)^{2}$

where $Q_{m A}, Q_{m B}$ - change of the energy at the melting; $Q_{v A}, Q_{v B}$ - change of the energy at the evaporation; $\sigma_{A}, \sigma_{B}-$ surface energy of pure components (in the liquid state); $z$ - coordination number; $z_{b}$ - number of the nearest neighbours of this atom situated in the neighboring layer; $x, y-$ concentrations of the component $\mathrm{B}$ in the liquid and solid phases, correspondingly; $N_{S}-$ number of atoms per unit of surface area in the layer parallel to the heterointerface [6].

Under $U>\Delta \mu$, the process of crystallization changes into the process of dissolution, as $\Delta \mu_{s t}$ becomes a negative value. Equality of $U\left(r^{\prime}\right)=\Delta \mu$ determines maximum allowed strain in two-dimensional nucleus of QD growing according to the Volmer Weber growth mechanism. In the assumption that elastic displacements $l_{a_{1}}=0, l_{a_{2}}=0$, modulus of shearing $G$ and lattice constants $a_{1}, a_{2}$ are not dependent on temperature, the maximal diameter of twodimensional nucleus of QD under these growth conditions is determined by the equation:

$d=2 r^{\prime}=2 \sqrt{\frac{\Delta \mu\left(a_{1}+a_{2}\right) a_{1} a_{2} N_{S}}{G\left(a_{1}-a_{2}\right)^{2}}}$.

Nuclei forming on the surface of the substrate have the form of the spherical segments with radius of curvature that corresponds to that of a homogenous critical nucleus formed in the liquid phase under the same conditions of crystallization. The calculation of radius of curvature was made by the expression:

$$
r^{*}=\frac{2 \sigma M}{\rho R T \ln \frac{C}{C_{0}}}
$$

where $R$ is the absolute gas constant, $\rho, M-$ density and molar mass of nucleus matter.

The lateral size of the basis of the nucleus is calculated in the assumptions that heteroepitaxial nucleus with the height of $h^{*}$ equal to monolayer thickness is pseudomorphous and have the radius of curvature $r^{*}$. Table 2 represents the diameters calculated at the process temperature in the range from 300 to $500{ }^{\circ} \mathrm{C}$ and supercooling $\Delta T_{F}$ in the range from 1 to $5^{\circ} \mathrm{C}$.

At the process of the QD nuclei growth the increase of the mechanical strain takes place in the layer of QD material, which abut on heterointerface. This increase passes till the values, corresponded to heterostructures GaAs - InAs with elastic constant of contacting materials in heterointerface. Strain in the QD has the gradient, directed normally to surface of the substrate. So, in the basis of QD the curvilinear front of etching of the QD side is forming. The curvilinearity of front changes the conditions of the local phase equilibrium, nearby the heterointerface as compared with the flat front of etching. This changing is described by the Gibbs - Thomson equation:

$\Delta \mu_{G T}=\Omega \sigma\left(R_{1}^{-1}+R_{2}^{-1}\right)$,

where $\Omega$ - specific volume of crystal phase; $R_{1}, R_{2}-$ the main radii of the interface curvature.

Size of the QD base is determined by the equation:

$$
d=\sqrt{\frac{4\left(\Delta \mu-\Delta \mu_{G T}\right)\left(a_{1}+a_{2}\right) a_{1} a_{2} N_{S}}{G\left(a_{1}-a_{2}\right)^{2}}} .
$$

The further growth of the nucleus is realized by the way of its normal growth within the period of the cooling pulse duration $\tau$. The rate of the growth of spherical nucleus in case of the isothermal decay of the supersaturated solution is determined by the Frenkel expression:

$\mathrm{v}=\frac{d r}{d t}=\frac{D}{N_{V} r}\left[\left(C-C_{0}\right)-\frac{b}{r}\right]$,

where $b=\frac{2 \sigma M C_{0}}{\rho R T} ; N_{V}-$ the number of atoms in the unit of volume of QD material [7].

Table 2. The lateral size (diameter) of the basis of the nucleus InAs on GaAs substrate.

\begin{tabular}{|c|c|c|c|c|c|}
\hline \multirow{2}{*}{$\begin{array}{c}\Delta T_{F}, \\
{ }^{\circ} \mathrm{C}\end{array}$} & \multicolumn{4}{|c|}{$\begin{array}{c}\text { Diameter of the basis of the nucleus } d, \mathrm{~nm}, \\
\text { for the various process temperatures }\end{array}$} \\
\cline { 2 - 6 } & $300^{\circ} \mathrm{C}$ & $350^{\circ} \mathrm{C}$ & $400^{\circ} \mathrm{C}$ & $450^{\circ} \mathrm{C}$ & $500^{\circ} \mathrm{C}$ \\
\hline 1 & 10.54 & 11.02 & 11.42 & 11.84 & 12.28 \\
\hline 2 & 7.40 & 7.72 & 8.04 & 8.34 & 8.64 \\
\hline 3 & 6.00 & 6.28 & 6.54 & 6.78 & 7.02 \\
\hline 4 & 5.18 & 5.40 & 5.62 & 5.82 & 6.04 \\
\hline 5 & 4.60 & 4.80 & 2.50 & 5.20 & 5.40 \\
\hline
\end{tabular}

Table 3. The height of InAs QD on GaAs substrate.

\begin{tabular}{|c|c|c|c|c|c|}
\hline \multirow{2}{*}{$\begin{array}{c}\Delta T_{F}, \\
{ }^{\circ} \mathrm{C}\end{array}$} & \multicolumn{5}{|c|}{ Height of QDe various process temperatures } \\
\cline { 2 - 6 } & $300{ }^{\circ} \mathrm{C}$ & $350{ }^{\circ} \mathrm{C}$ & $400{ }^{\circ} \mathrm{C}$ & $450{ }^{\circ} \mathrm{C}$ & $500{ }^{\circ} \mathrm{C}$ \\
\hline 1 & 4.40 & 8.21 & 13.98 & 22.07 & 32.77 \\
\hline 2 & 6.23 & 11.68 & 19.91 & 31.45 & 46.72 \\
\hline 3 & 7.59 & 14.25 & 24.32 & 38.46 & 57.18 \\
\hline 4 & 8.70 & 16.37 & 27.97 & 44.27 & 65.86 \\
\hline 5 & 9.65 & 18.18 & 31.09 & 49.24 & 73.30 \\
\hline
\end{tabular}




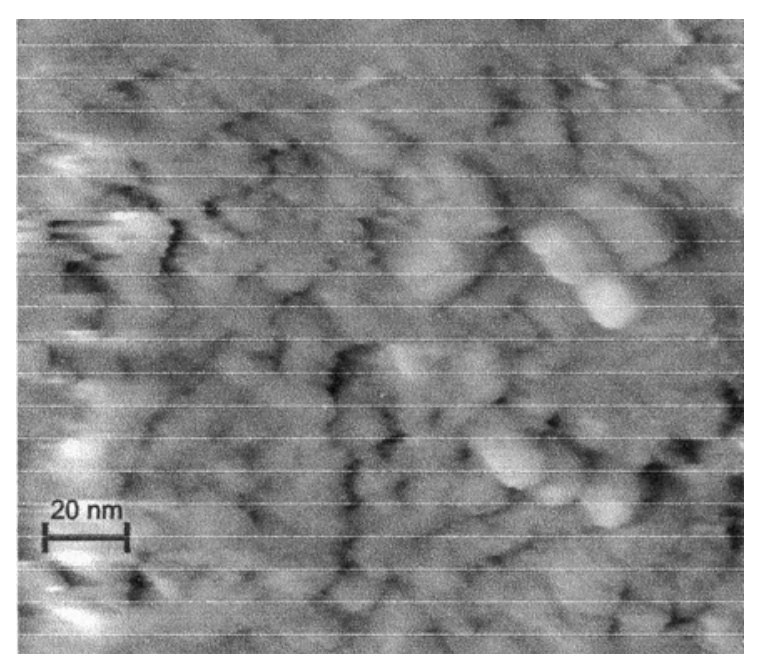

Fig. 2. STM-image of uncovered QD $(158 \times 180 \mathrm{~nm})$.

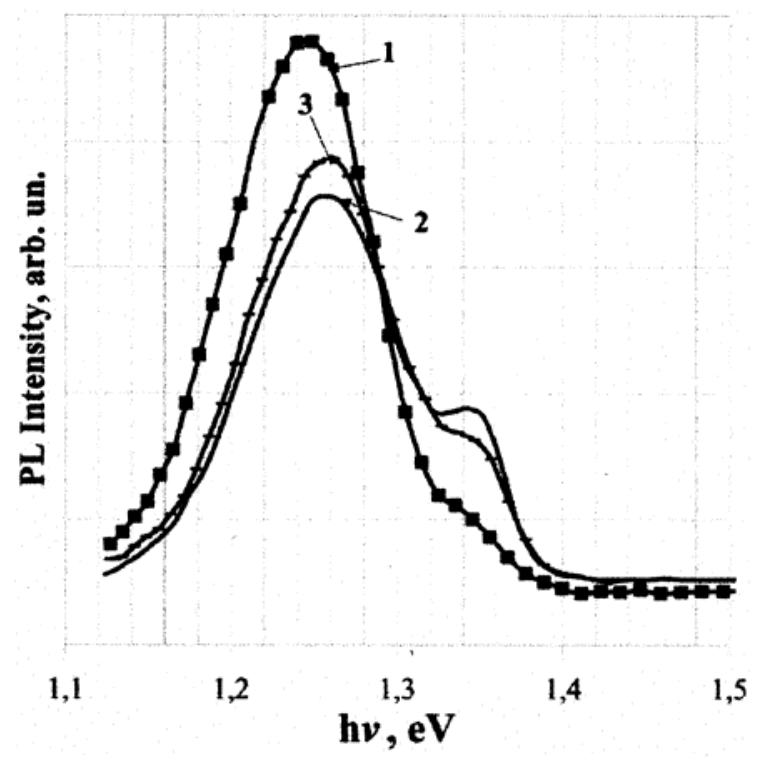

Fig. 3. PL spectra of the structure with uncovered QDs: 1 semiconductor laser (1:1), 2 - helium-neon (1:10), 3 - argon $(1: 10)$.

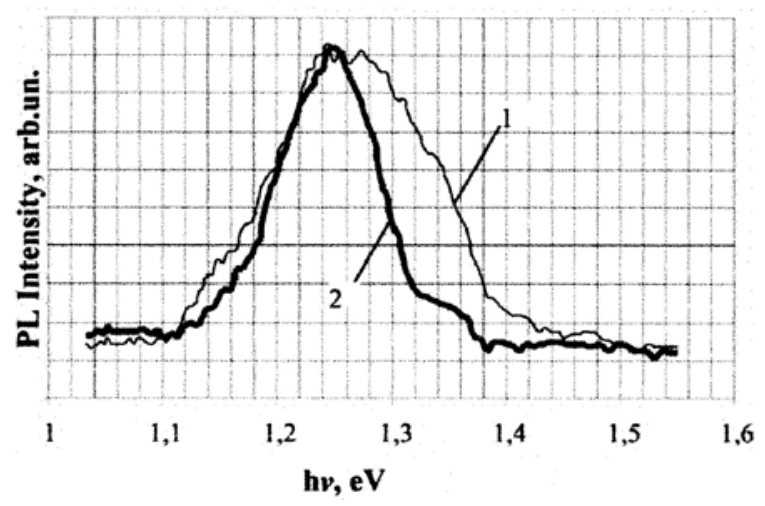

Fig. 4. PL spectra of samples: 1 - disoriented by 4 from (100) substrate, 2 - oriented (100) substrate.
By using the averaged values of supercooling and concentration of solution during the pulse, the height of nucleus depending on the pulse length was estimated by the equation:

$h=\sqrt{\left(h^{*}\right)^{2}+\frac{2}{5} \frac{D}{N_{V}}\left(C-C_{0}\right) \tau}$.

The results of calculation of the height of InAs QD on GaAs at different values of the process temperature $T_{1}$ are represented in Table 3.

The essential feature of the QD formation process is the thermodynamic instability of the crystallization front caused by the difference in properties of substrate and QD materials. In this connection, after bringing the substrate surface into a contact with solution-melt, the period of QD formation should be minimized.

\section{Results and discussion}

STM-image of QDs uncovered by a matrix layer and grown on GaAs substrate surface with orientation (100) at $T_{1}=400{ }^{\circ} \mathrm{C}, \Delta T_{F}=5{ }^{\circ} \mathrm{C}$ and $\delta=0.3 \mathrm{~cm}$ from solution in indium melt is represented in Fig. 2.

The PL spectra of this sample (N 1) at $77 \mathrm{~K}$ have a maximum, position of which changes in the range $1.24<h v<1.26 \mathrm{eV}$ (Fig. 3), and its intensity rises by 7 times on condition that the power of exciting radiation increases from 5 up to $20 \mathrm{~mW}$. At the further increase of the power up to $2 \mathrm{~W}$, the changes in the position and in the PL intensity of this maximum are negligible.

The same maximum was also seen in PL spectra of samples of multilayer structures (structures with vertically bound QD) containing two, three, four, six, and ten layers of QD as well as nanosize (3-5 nm) layers of GaAs located between dots and over their surface, fabricated in the conditions when the sample $\mathrm{N} 1$ was obtained. The identity of the PL spectra at $77 \mathrm{~K}$ of the structures with uncovered QD and with QD covered by nanosize GaAs layers testifies to that nanosize layers of GaAs do not result to QD deformation or to changes in its structure.

Fig. 4 shows PL spectra of two samples at $77 \mathrm{~K}$, which contains a system of vertically bound InAs dots. One of them has been grown on GaAs substrate disoriented by $4^{\circ}$ from (100) in $<110>$ direction (curve 1), another one - on a substrate with surface orientation (100). The match of the longwave slopes of PL spectra of these samples testifies that the maximum sizes of QD in them are identical, and shortwave range shift of the shortwave slope of the emission spectrum of one of the samples observed (curve 1) testifies to the presence of QDs with smaller sizes in this sample. Formation of such QD may be conditioned by that the substrate surface of this sample includes stages formed by flat terraces oriented parallel to (100) plane and by butt ends of the stages oriented parallel to (111) plane. Since the width of the butt ends of stages is much less than the width of terraces of the stages, QD formed on 
the butt ends have smaller sizes than the size of those on the terraces of stages.

The thickness of matrix material layer influenced essentially on the PL maximum location in structures with QD. Thus, in PL spectra of heterostructures with one QD layer grown in the same conditions as sample $\mathrm{N} 1$, but covered by GaAs layer with thickness more than $0.2 \mu \mathrm{m}$, the maximum of emission was observed at $h v=1.35 \mathrm{eV}$ and a weak maximum at $h v=1.24 \mathrm{eV}$. Originating of the maximum at $h v=1.35 \mathrm{eV}$ in such layers may be connected to formation of a quantumdimensional layer of solid solution in QD close to heterointerface "QD - covering layer" or to deformation of QD material close to heterointerface with GaAs.

Wide bands in the range of 1.05 to $1.2 \mathrm{eV}$ were observed at $300 \mathrm{~K}$ in PL spectra of all the multilayer structures investigated, and with increase of a number of vertically bound QDs the spectrum shifted into the longwave area.

\section{Conclusion}

The represented results of investigations show that the method of pulse cooling of saturated solution melt allows to fulfilled the growth of heterostructures with QDs. The QD sizes are determined by the supercooling of the crystallization front, duration of cooling pulse and value of the surface energy of the solid phase. The QD sizes determined from the analyses of photoluminescence spectra are in accord with the data, which is found using the analysis of STM-image of structures with uncovered QDs.

\section{Acknowledgments}

The researches described in this publication were possible partly by Grant Award \# UE2-2225 of the U.S. Civilian Research and Development Foundation for the Independent States of the Former Soviet Union (CRDF). The authors also thank Yu. Jegerya for valuable contribution to obtaining the STM-images of QDs.

\section{References}

1. V.P. Dragunov, I.G. Neizvestny, V.A. Gridshin Fundamentals of nanoelectronics, NSTU Printing, Novosibirsk, p. 332 (2000).

2. N.N. Ledentsov, V.M. Ustinov, V.A. Shchukin, P.S. Kop'ev, Z.I. Alferov, D. Bimberg, Heterostructures with quantum dots: obtaining, properties, lasers. Review // Fiz. Tekhn. Poluprov. 32, p. 4 (1998).

3. T.F. Kulutkina, I.E. Maronchuk, A.V. Shorokhov, Growing of submicron layers during pulse cooling of saturated solution-melt // Techn. Phys. Lett. 21, p. 20 (1995).

4. A.A. Chernov, Crystallization processes // Modern crystallography 3, p. 7-230, Nauka, Moscow (1980).

5. J.H. van der Merwe // J. Appl. Phys. 34, p. 117-127.

6. P.A. Rehbinder, Surface phenomena in dispersed systems // Physicochemical mechanic, Nauka, Moscow (1979).

7. Y.I. Frenkel, The introduction into the theory of metals, Nauka, Leningrad (1972). 